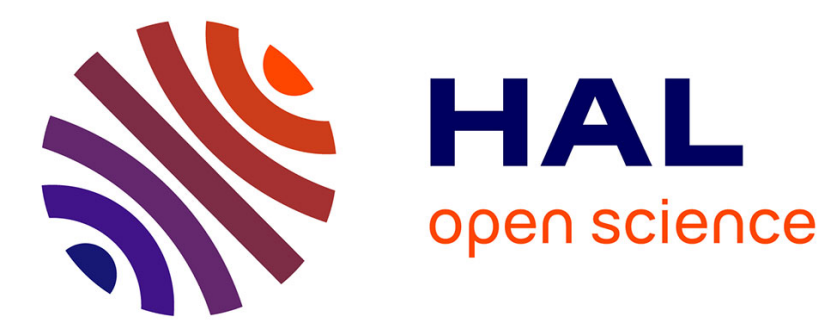

\title{
Resummation of diagrammatic series with zero convergence radius for strongly correlated fermions
}

Riccardo Rossi, Takahiro Ohgoe, Kris van Houcke, Felix Werner

\section{To cite this version:}

Riccardo Rossi, Takahiro Ohgoe, Kris van Houcke, Felix Werner. Resummation of diagrammatic series with zero convergence radius for strongly correlated fermions. Phys.Rev.Lett., 2018, 121 (13), pp.130405. 10.1103/PhysRevLett.121.130405 . hal-01730049

\section{HAL Id: hal-01730049 \\ https://hal.science/hal-01730049}

Submitted on 20 Jan 2022

HAL is a multi-disciplinary open access archive for the deposit and dissemination of scientific research documents, whether they are published or not. The documents may come from teaching and research institutions in France or abroad, or from public or private research centers.
L'archive ouverte pluridisciplinaire HAL, est destinée au dépôt et à la diffusion de documents scientifiques de niveau recherche, publiés ou non, émanant des établissements d'enseignement et de recherche français ou étrangers, des laboratoires publics ou privés. 


\title{
Resummation of Diagrammatic Series with Zero Convergence Radius for Strongly Correlated Fermions
}

\author{
R. Rossi, ${ }^{1, *}$ T. Ohgoe, ${ }^{2}$ K. Van Houcke, ${ }^{1}$ and F. Werner ${ }^{3}$ \\ ${ }^{1}$ Laboratoire de Physique Statistique, Ecole Normale Supérieure-Université PSL, CNRS, \\ Sorbonne Université, Université Paris Diderot, 75005 Paris, France \\ ${ }^{2}$ Department of Applied Physics, University of Tokyo, 7-3-1 Hongo, Bunkyo-ku, Tokyo 113-8656, Japan \\ ${ }^{3}$ Laboratoire Kastler Brossel, Ecole Normale Supérieure-Université PSL, CNRS, \\ Sorbonne Université, Collège de France, 75005 Paris, France
}

(Received 21 February 2018; revised manuscript received 26 July 2018; published 27 September 2018)

\begin{abstract}
We demonstrate that a summing up series of Feynman diagrams can yield unbiased accurate results for strongly correlated fermions even when the convergence radius vanishes. We consider the unitary Fermi gas, a model of nonrelativistic fermions in three-dimensional continuous space. Diagrams are built from partially dressed or fully dressed propagators of single particles and pairs. The series is resummed by a conformal-Borel transformation that incorporates the large-order behavior and the analytic structure in the Borel plane, which are found by the instanton approach. We report highly accurate numerical results for the equation of state in the normal unpolarized regime, and reconcile experimental data with the theoretically conjectured fourth virial coefficient.
\end{abstract}

DOI: 10.1103/PhysRevLett.121.130405

Feynman diagrams are a powerful computational tool and have led to an impressive list of important approximate results in various branches of physics. But is it possible to make accurate predictions by summing up Feynman diagrams?

The answer is certainly yes if the coupling constant is small. The most famous example is quantum electrodynamics. Dyson argued that the vacuum becomes unstable at negative fine-structure constant and hence the convergence radius should be zero [1]. Nonetheless, thanks to the smallness of the coupling constant, the diagrammatic series behaves as a convergent series for all practical purposes, leading to the most stringently tested physical theory [2-4].

But what about strongly correlated theories? In the pioneering work [5-7] critical exponents were accurately computed by summing up Feynman diagrams in the strongly correlated regime of $\phi^{4}$ theory. The problem of zero convergence radius was overcome by computing the large-order asymptotic behavior [8,9] and using it to build an appropriate resummation technique based on a conformal-Borel transformation.

For fermions on a lattice, it is known in the mathematical physics literature that the convergence radius of diagrammatic series is nonzero in some part of the phase diagram [10-12]. In recent years, this led to controlled thermodynamic-limit results in correlated regimes by summing up convergent diagrammatic series to high enough order using the diagrammatic Monte Carlo approach [13-21], where the fermionic sign plays a very different role than in conventional quantum Monte Carlo methods [22].

In this Letter, we report high-precision results obtained by summing up Feynman-diagram series for a strongly correlated continuous-space fermionic theory with zero convergence radius. Specifically, we consider non-relativistic spin- $1 / 2$ fermions in three space dimensions with contact interaction-a model which accurately describes ongoing ultracold atom experiments and is also relevant to neutron matter [23-33]. We derive the large-order asymptotic behavior of the diagrammatic series, and we give mathematical arguments and numerical evidence for the resummability of the series by a specifically designed conformal-Borel transformation that incorporates the large-order behavior and the knowledge of the analytical structure standing behind the series. Combining this new resummation method with diagrammatic Monte Carlo evaluation up to order 9 , we obtain new results for the equation of state in the normal phase, which agree with the ultracold-atom experimental data from Refs. [25,26], except for the 4th virial coefficient for which our data point to the theoretically conjectured value of Ref. [34].

In order to have a well-defined diagrammatic framework for the contact interaction in continuous space, it is necessary to incorporate exactly the two-particle scattering problem. This is done most naturally by using the sum of all ladder diagrams $\Gamma_{0}$ as an effective interaction vertex between $\uparrow$ and $\downarrow$ fermions, or equivalently, a partially dressed pair propagator. Diagrammatically,

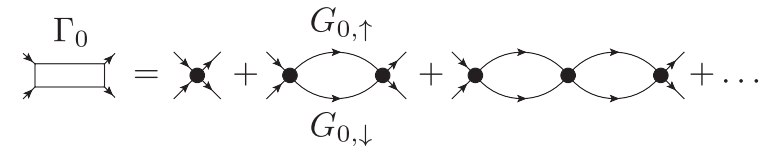


where the - denotes the bare coupling constant and $G_{0}$ is the free fermion propagator, given by $G_{0, \sigma}\left(p, \omega_{\nu}\right)=$ $\left(i \omega_{\nu}+\mu_{\sigma}-p^{2} / 2 m\right)^{-1}$ in the momentum Matsubarafrequency representation. Here, $\sigma \in\{\uparrow, \downarrow\}, \mu_{\sigma}$ is the chemical potential, $m$ the fermion mass, and $\omega_{\nu}=(2 \nu+1) \pi / \beta$ with $\beta=1 /\left(k_{B} T\right)$ the inverse temperature. $\Gamma_{0}$ is well defined for the continuous-space zero-range interaction (without momentum cutoff) and only depends on the $s$-wave scattering length $a$ (apart from $\mu_{\uparrow}, \mu_{\downarrow}, \beta$, and external momentum frequency). The same property holds for higher-order diagrams built from $G_{0}$ and $\Gamma_{0}$. This "ladder scheme" is suited to describe the crossover between Fermi and Bose gases, and its lowest-order approximation (left diagram in Fig. 1) is widely used [33,35,36]. A diagrammatic Monte Carlo algorithm [13] allows us to stochastically evaluate all Feynman diagrams up to order 9 (see Fig. 1).

An intensive quantity $Q$, such as pressure or self-energy, can be formally written as a diagrammatic series $\sum_{N=0}^{\infty} a_{N}$. Here $a_{N}$ is a sum of connected diagrams of order $N$ (see Fig. 1). As we shall see this diagrammatic series is divergent, and it is not obvious how to give a meaning to the formal expansion $Q \stackrel{?}{=} \sum_{N=0}^{\infty} a_{N}$. To do so, we introduce a function $Q(z)$ whose Taylor series is $\sum_{N=0}^{\infty} a_{N} z^{N}$, and such that $Q(z=1)$ is the desired exact physical result. Here $z$ is a formal parameter playing the role of an effective coupling constant. A nonperturbative construction of $Q(z)$ is realized by introducing the action [37]

$$
\begin{aligned}
S^{(z)}= & -\int d^{3} r \int_{0}^{\beta} d \tau\left(\sum_{\sigma=\uparrow, \downarrow} \bar{\varphi}_{\sigma} G_{0, \sigma}^{-1} \varphi_{\sigma}+\bar{\eta} \Gamma_{0}^{-1} \eta\right. \\
& \left.-z \bar{\eta} \Pi_{0} \eta+\sqrt{z}\left(\bar{\eta} \varphi_{\downarrow} \varphi_{\uparrow}+\bar{\varphi}_{\uparrow} \bar{\varphi}_{\downarrow} \eta\right)\right),
\end{aligned}
$$

where $\varphi_{\sigma}$ are fermionic Grassmann fields, $\eta$ is a bosonic complex field, and $\Pi_{0}$ is the particle-particle bubble $\left(G_{0, \uparrow} G_{0, \downarrow}\right)$, which cancels out all diagrams containing particle-particle bubbles, as required to avoid double counting. For example, for the pressure we simply have

$$
Q(z)=\lim _{\mathcal{V} \rightarrow \infty} \frac{1}{\beta \mathcal{V}} \ln \int \mathcal{D} \varphi \mathcal{D} \eta e^{-S^{(z)}[\varphi, \eta]}
$$

with $\mathcal{V}$ the volume.
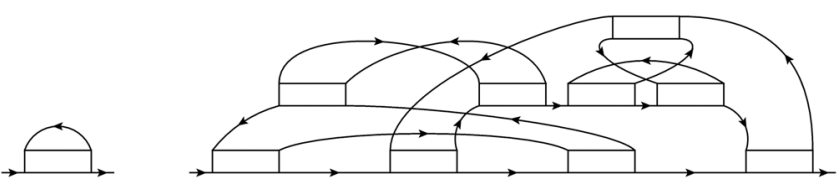

FIG. 1. First-order diagram (left) and an example of 9th-order diagram (right) for the single-particle self-energy. Each line represents a single-particle propagator while each box is a bosonic pair propagator.
Large-order behavior.-We now turn to the crucial problem of computing the large- $N$ behavior of $a_{N}$. In the pioneering works [8,9], the large-order behavior for $\phi^{4}$ theory was obtained from a saddle point of the functional integral. To study the large-order behavior of fermionic theories, it was found essential to integrate out fermionic fields, which leads to a purely bosonic functional integral, whose integrand $e^{-S_{\text {eff }}^{(z)}[\eta]}$ can be estimated in the large-field limit using a Thomas-Fermi (i.e., quasilocal) approximation [38-42]. In our problem, this procedure can be justified by showing that this integrand is an entire function of $z$ $[43,44]$. We find that the bosonic action $S_{\text {eff }}^{(z)}[\eta]$ scales as $z^{5 / 4} \int d^{3} r d \tau|\eta(\mathbf{r}, \tau)|^{5 / 2}$ for large $|\eta|$. The saddle-point method then gives

$$
\begin{aligned}
a_{N}= & \Gamma(N / 5) A^{-N} \\
& \times \operatorname{Re} \exp \left[i 4 \pi N / 5-U_{1} e^{i \pi / 5} N^{4 / 5}+O\left(N^{3 / 5}\right)\right],
\end{aligned}
$$

where $U_{1}=5^{1 / 5} A$ and

$A:=\frac{1}{\pi^{2}}\left(\frac{4}{5 \Gamma(3 / 4)^{8}}\right)^{\frac{1}{5}} \min _{\eta} \frac{-\left\langle\eta\left|\Gamma_{0}^{-1}\right| \eta\right\rangle}{\left(\int d^{3} r d \tau|\eta(\mathbf{r}, \tau)|^{5 / 2}\right)^{4 / 5}}$.

The fact that $a_{N}$ is of order $(N !)^{1 / 5}$ immediately implies that the radius of convergence is zero. This raises a fundamental question: Can the exact physical result still be constructed in a unique way from the set $\left\{a_{N}\right\}$ ?

Resummation.-Given the above asymptotic behavior, it is natural to introduce the generalized Borel transform defined by

$$
\begin{aligned}
B(z) & :=\sum_{N=0}^{\infty} \frac{a_{N}}{\mu_{N}} z^{N}, \quad|z|<A, \\
\mu_{N} & :=\int_{0}^{\infty} d t t^{4} e^{-t^{5}-b t^{4}-c t^{3}} t^{N} .
\end{aligned}
$$

Note that $\mu_{N} \sim \Gamma(N / 5) \exp \left[-b(N / 5)^{4 / 5}\right]$ for $N \rightarrow \infty$. The corresponding inverse Borel transformation reads

$$
Q_{B}(z):=\int_{0}^{\infty} d t t^{4} e^{-t^{5}-b t^{4}-c t^{3}} B(z t),
$$

where $b$ and $c$ are free parameters at this stage.

The answer to the above unicity question is then given by the following theorem due to Nevanlinna [45-49]. Let $W:=\{z \in \mathbb{C}|0<| z|<R,| \arg z \mid<\pi / 10+\epsilon\}$, for some $R>0$ and $\epsilon>0$. If

(1) $Q(z)$ is analytical for $z \in W$,

(2) $\exists \tilde{A}$ and $C$ such that $\left|d^{N} Q(z) / d z^{N}\right| / N ! \leq C \tilde{A}^{-N}(N \text { ! })^{1 / 5}$ for all $N \geq 0$ and $z \in W$,

(3) $a_{N}=\lim _{z \rightarrow 0, z \in W} d^{N} Q(z) / d z^{N} / N$ ! then

(i) $B(z)$ can be analytically continued for $z \in \mathbb{R}_{+}$,

(ii) $\exists R^{\prime}>0$ such that $Q_{B}(z)=Q(z)$ for $z \in\left[0, R^{\prime}\right]$. 
The hypotheses of this theorem hold in our situation for the following reasons: Hypothesis 1 follows from the functional integral representation (2) and the fact that the integrand, after integrating out the fermions, is an entire function of $z$ that can be bounded in the large- $\eta$ limit using the Thomas-Fermi result. Hypothesis 2 can be obtained in a similar way to the large-order behavior of $a_{N}$. Hypothesis 3 is plausible given that the functional integral for $z \in W$ is absolutely convergent.

The problem of resummation is thereby reduced to the one of analytical continuation of the Borel transform $B(z)$ to the whole real positive axis. To this end, it is essential to know the analytical structure of $B(z)$ in the complex $z$ plane. As follows from the large-order behavior of $a_{N}, B(z)$ has singularities at $z_{+}=A \exp ( \pm i 4 \pi / 5)$. We find that $B(z)$ can be analytically continued to $\mathcal{D}:=\mathbb{C} \backslash\{z \in \mathbb{C}|| \arg z \mid=$ $4 \pi / 5,|z| \geq A\}$ under the condition $|\theta(b)|<\pi / 5$, where $\theta(b):=\arg \left[\exp (i \pi / 5) U_{1}-b / 5^{4 / 5}\right]$ (note that $b=0$ is not allowed). This analytical continuation is explicitly realized by the one-to-one conformal transformation $h$ that maps the open unit disc $\mathcal{B}$ onto $\mathcal{D}$ such that $h(0)=0$ and $[h(w)]^{*}=$ $h\left(w^{*}\right)$; see Fig. 2. In practice, the function $\tilde{B}(w):=$ $B[h(w)]$, a priori defined for $|h(w)|<A$, has a Taylor series $\sum_{N=0}^{\infty} \tilde{B}_{N} w^{N}$ that converges for all $w \in \mathcal{B} ; B(z)$ can then be computed as $\tilde{B}\left[h^{-1}(z)\right]=\sum_{N=0}^{\infty} \tilde{B}_{N}\left[h^{-1}(z)\right]^{N}$ for all $z \in \mathcal{D}$. Substituting this expression into the inverse Borel transform (8) finally yields $Q(1)=$ $\lim _{N_{\max } \rightarrow \infty} \sum_{N=0}^{N_{\max }} B_{N}^{\left(N_{\max }\right)} a_{N}$ where the coefficients $B_{N}^{\left(N_{\max }\right)}$ are computed easily thanks to the expression of the conformal mapping

$$
h(w)=\frac{4^{\frac{9}{5}} A w}{5(1+w)^{2 / 5}(1-w)^{8 / 5}} .
$$

We compute $A$ from Eq. (5) by numerical minimization; the minimizer $\eta_{c}$ can be called instanton, or more appropriately soliton since it can be shown to be independent of imaginary time (it is also rotationally invariant and spatially localized). We note that $Q(z)$ has the two branch cuts $\{\arg z= \pm 4 \pi / 5\}$, along which it has discontinuities

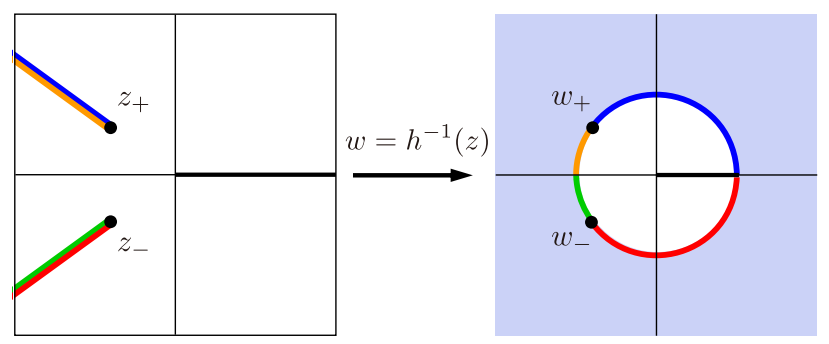

FIG. 2. Conformal mapping: the singularities of the Borel transform (in color) are mapped onto the unit circle. The two points $z_{ \pm}$are mapped onto $w_{ \pm}$and the real positive axis is mapped onto the segment $[0,1]$. $\sim e^{-(A /|z|)^{5}}$ for $|z| \rightarrow 0$ (this follows from the analyticity of $B(z)$ in $\mathcal{D}$ and the large-order behavior of $a_{N}$ ) [50].

Bold scheme.-In order to access lower temperatures, we turn to the "bold scheme" where diagrams are built selfconsistently from fully dressed propagators. While in the above ladder scheme, lines and boxes in Fig. 1 denoted $G_{0}$ and $\Gamma_{0}$, in the bold scheme they denote the fully dressed $G$ and $\Gamma$ [60]. Starting from an action $S_{\text {bold }}^{(z)}$ constructed as in Ref. [37], we find the large-order behavior (4) modulo the replacement of $\Gamma_{0}$ by $\Gamma$ in Eq. (5) and a modified expression for $U_{1}[43,44]$. The justification is less solid than in the ladder scheme, because the integrand of the purely bosonic functional integral is not entire in $z$. The self-consistent computation is done as follows: Starting from some initial guess for $G$ and $\Gamma$, we use the bold diagrammatic Monte Carlo algorithm described in Ref. [60] to compute the (skeleton) diagrammatic series for the single-particle self-energy $\Sigma$ and the pair self-energy $\Pi$. We then apply the conformal-Borel resummation procedure to these diagrammatic series. The resulting resummed $\Sigma$ and $\Pi$ are then plugged into the Dyson equations to obtain new propagators $G$ and $\Gamma$. This cycle is repeated until convergence [61].

We note that on approach to the superfluid transition, $A \rightarrow 0$ so that the series becomes increasingly hard to resum, while in the high-temperature limit, $A \rightarrow \infty$ (for both ladder and bold schemes) so that the series divergence becomes weaker.

Numerical results. - In this Letter we focus on the central point of the BEC-BCS crossover, the unitary limit, where the dimer binding energy vanishes and the scattering length diverges. This unitary Fermi gas is strongly correlated since the scattering cross section is on the order of the squared interparticle distance. We report results for the equation of state (EOS) in the normal phase, restricting for now to the unpolarized gas, $\mu=\mu_{\uparrow}=\mu_{\downarrow}$. Scale invariance implies than the rescaled density $n \lambda^{3}$ is a universal function of $\beta \mu$, with $\lambda=\sqrt{2 \pi \hbar^{2} \beta / m}$ the thermal wavelength.

In the moderately degenerate regime, we find very good convergence of the series as a function of the maximal diagram order $N_{\max }$ after resummation by the new conformal-Borel transformation, see Fig. 3. The final results for ladder and bold schemes agree within their error bars which are below $0.1 \%$. The value measured at MIT is $2 \%$ higher, a deviation within the experimental uncertainty [26].

Here and in what follows we empirically fixed the free parameter $b$ such that $\theta(b)=\pi / 10$ (i.e., $b=-5^{4 / 5} U_{1}$ ). We observed consistent results for different values of the free parameter $c$, and we adjusted it to optimize the convergence. In Fig. 3, the conformal-Borel transformation was applied to $Q(z)=n(z)$ with $c=12$ for the ladder scheme, and $Q(z)=\Sigma(z) / z$ respectively $\Pi(z) / z$ with $c=10$ for the bold scheme [64]. The error bars shown at each $N_{\max }$ include the statistical noise coming from the Monte Carlo, 


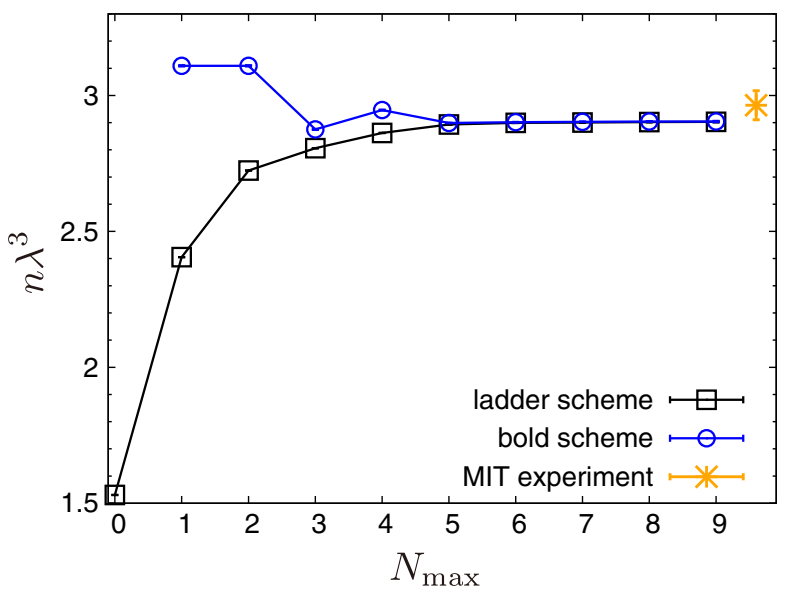

FIG. 3. Resummed density vs maximal diagram order at $\beta \mu=0 \quad\left(T / T_{F} \approx 0.6\right)$. The ladder and bold diagrammatic schemes agree with each other and with experiment.

and for the bold scheme also the error due to the finite number of iterations. Our final error bars also include errors due to finite $N_{\max }$ and to cutoffs and discretizations in the numerics, so that all sources of errors are taken into account [65].

At lower temperatures, the ladder scheme is not applicable (due to a pole in $\Gamma_{0}$ ) but we still observe convergence of the bold scheme, as shown in Fig. 4, where we crosscheck three variants of the conformal-Borel resummation: $Q(z)=\Sigma(z) / z$ respectively $\Pi(z) / z$ with $c=13$ (circles), the same $Q(z)$ with $c=60$ (diamonds), and $Q(z)=\Sigma(z)$ respectively $\Pi(z)$ with $c=60$ (squares). Our final result agrees with the MIT measurement up to a $3 \%$ deviation consistent with the experimental uncertainty.

In the related earlier work [67], much simpler resummation methods such as the Lindelöf method were used, assuming that the diagrammatic series has a nonzero

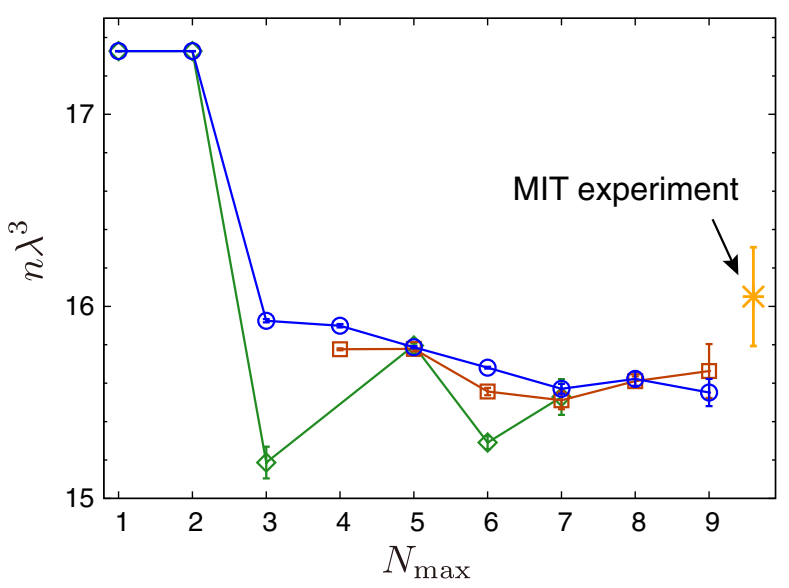

FIG. 4. Density vs maximal diagram order at $\beta \mu=2$ $\left(T / T_{F} \approx 0.2\right)$. The bold diagrammatic series is resummed by three variants of the conformal-Borel transformation (see text). convergence radius. This assumption is invalidated by the large-order behavior $\left|a_{N}\right| \sim(N !)^{1 / 5}$ found here. Hence the results of Ref. [67] contained a systematic error. Nevertheless, they deviate from the new results reported here by less than $2 \%$, which is likely related to the smallness of the exponent $1 / 5$.

The subfactorial scaling $\left|a_{N}\right| \sim(N !)^{1 / 5}$ also implies that for a given order $N$, the sum $a_{N}$ of all diagrams is much smaller than the number $\sim N$ ! of diagrams. This is a manifestation of the massive cancellation between different diagrams due to the fermionic sign.

Finally, we turn to the higher-temperature regime, where our new high-accuracy data shed light on a controversy. In the limit $T \gg T_{F}$, the EOS admits a virial expansion $n_{\text {virial }}^{(J)} \lambda^{3}=2 \sum_{j=0}^{J} j b_{j} \zeta^{j}$ in powers of the fugacity $\zeta=e^{\beta \mu}$. The virial coefficient $b_{j}$ is determined by the $j$-body problem, and is known exactly for $j=2[23,68]$ and $j=3[69,70]$. In Fig. 5 we subtract the known virial-3 result from our EOS data so that the result tends to $b_{4}$ in the nondegenerate limit $\zeta \rightarrow 0$. Accordingly, we display at $\zeta=0$ several values reported for $b_{4}$ : The value obtained by Endo and Castin [34] (based on a physically motivated mathematical conjecture) deviates from the values reported by experimentalists from ENS [25] and MIT [26]. The dedicated path integral quantum Monte Carlo result of Yan and Blume [71] has an error bar too large to resolve the discrepancy. Our data suggest that the Endo-Castin result is correct, but requires sufficiently small $\zeta$ to be extracted, and correspondingly high accuracy to resolve the difference $n-n_{\text {virial }}^{(3)} \propto \zeta^{4}$ (at $\zeta \approx 0.2$ our error on $n \lambda^{3}$ is $<0.01 \%$ ), while extrapolations from $\zeta \gtrsim 0.6$ lead to the overestimated $b_{4}$ values reported in Refs. [25,26]. In other words, at $\zeta \approx 0.6\left(T / T_{F} \approx 1\right)$ the unitary Fermi gas is still so strongly correlated that it cannot be reduced to a 4-body problem.

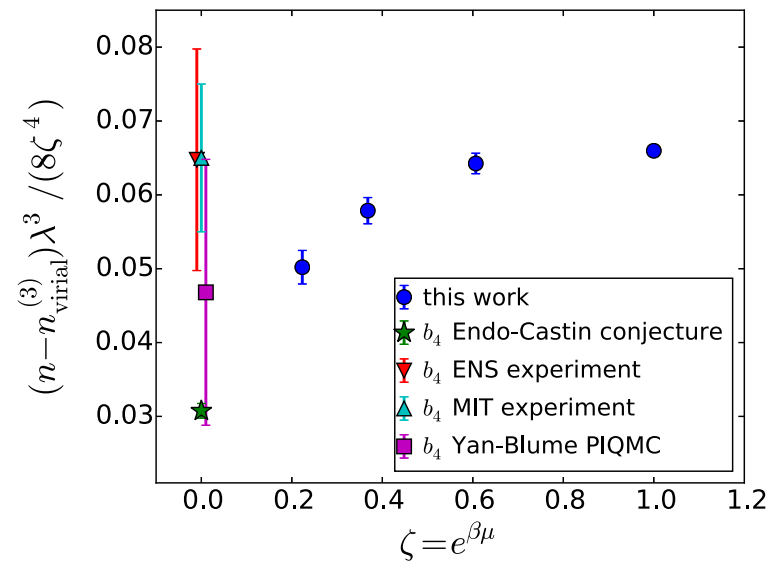

FIG. 5. Equation of state and 4th virial coefficient: The difference between the density $n$ and its 3rd order virial expansion $n_{\text {virial }}^{(3)}$, divided by the appropriate factor, must tend to the 4 th virial coefficient $b_{4}$ in the high-temperature limit $\zeta \rightarrow 0$. 
In summary, we found that for the unitary Fermi gas, a strongly correlated fermion model without small expansion parameter, diagrammatic series built on partially or fully dressed propagators can be Borel resummed and yield accurate unbiased results, even though the convergence radius is zero.

How does this relate to other fermionic theories? For QED, the situation is opposite: Large-order behavior and Borel summability are still open problems [40,41,56,72] but no resummation is needed in practice because the coupling constant is small. QCD combines both difficulties: It is nonperturbative and probably not Borel summable [57,73], which calls for new ideas [74]. The present approach may, however, be directly generalizable to other continuous-space strongly correlated fermion problems, such as nuclear matter or the electron gas.

We thank E. Brézin, Y. Castin, and B. Svistunov for stimulating discussions, and $\mathrm{M}$. $\mathrm{Ku}$ for providing the experimental data of Ref. [26]. F. W. was financially supported by ERC (Grants Thermodynamix and Critisup2), and T.O. by the MEXT HPCI Strategic Programs for Innovative Research (SPIRE), the Computational Materials Science Initiative (CMSI) and Creation of New Functional Devices and HighPerformance Materials to Support Next Generation Industries (CDMSI), and by a Grant-in-Aid for Scientific Research (No. 22104010, No. 22340090, No. 16H06345, and No. 18K13477) from MEXT, Japan. Simulations ran on the clusters "ponyo" at LKB-LPTMC/UPMC, "sveta" at UMass, "blue gene" at IDRIS, "curie" at TGCC, "MesoPSL," the K computer provided by the RIKEN Advanced Institute for Computational Science under the HPCI System Research project (Projects No. hp130007, No. hp140215, No. hp150211, No. hp160201, and No. hp170263), and at the Supercomputer Center, Institute for Solid State Physics, University of Tokyo.

*Present address: Center for Computational Quantum Physics, The Flatiron Institute, New York, USA.

[1] F. Dyson, Phys. Rev. 85, 631 (1952).

[2] T. Aoyama, M. Hayakawa, T. Kinoshita, and M. Nio, Nucl. Phys. B796, 184 (2008).

[3] R. Bouchendira, P. Cladé, S. Guellati-Khélifa, F. Nez, and F. Biraben, Phys. Rev. Lett. 106, 080801 (2011).

[4] D. Hanneke, S. Fogwell, and G. Gabrielse, Phys. Rev. Lett. 100, 120801 (2008).

[5] J. C. Le Guillou and J. Zinn-Justin, Phys. Rev. Lett. 39, 95 (1977).

[6] J. C. Le Guillou and J. Zinn-Justin, Phys. Rev. B 21, 3976 (1980).

[7] R. Guida and J. Zinn-Justin, J. Phys. A 31, 8103 (1998).

[8] L. N. Lipatov, Zh. Eksp. Teor. Fiz. 72, 411 (1977) [Sov. Phys. JETP 45, 216 (1977)].

[9] E. Brézin, J. C. Le Guillou, and J. Zinn-Justin, Phys. Rev. D 15, 1544 (1977).
[10] G. Benfatto, A. Giuliani, and V. Mastropietro, Ann. Henri Poincaré 7, 809 (2006).

[11] A. Giuliani and V. Mastropietro, Phys. Rev. B 79, 201403 (2009).

[12] V. Mastropietro, J. Phys. A 47, 465003 (2014).

[13] K. Van Houcke, E. Kozik, N. Prokof'ev, and B. Svistunov, Phys. Procedia 6, 95 (2010).

[14] E. Kozik, K. Van Houcke, E. Gull, L. Pollet, N. Prokof'ev, B. Svistunov, and M. Troyer, Europhys. Lett. 90, 10004 (2010).

[15] S. A. Kulagin, N. Prokof'ev, O. A. Starykh, B. Svistunov, and C. N. Varney, Phys. Rev. Lett. 110, 070601 (2013).

[16] Y. Deng, E. Kozik, N. V. Prokof'ev, and B. V. Svistunov, Europhys. Lett. 110, 57001 (2015).

[17] A. S. Mishchenko, N. Nagaosa, and N. Prokof'ev, Phys. Rev. Lett. 113, 166402 (2014).

[18] J. Gukelberger, E. Kozik, L. Pollet, N. Prokof'ev, M. Sigrist, B. Svistunov, and M. Troyer, Phys. Rev. Lett. 113, 195301 (2014).

[19] Y. Huang, K. Chen, Y. Deng, N. Prokof'ev, and B. Svistunov, Phys. Rev. Lett. 116, 177203 (2016).

[20] I. S. Tupitsyn and N. V. Prokof'ev, Phys. Rev. Lett. 118, 026403 (2017).

[21] W. Wu, M. Ferrero, A. Georges, and E. Kozik, Phys. Rev. B 96, 041105 (2017).

[22] R. Rossi, N. Prokof'ev, B. Svistunov, K. Van Houcke, and F. Werner, Europhys. Lett. 118, 10004 (2017).

[23] S. Giorgini, L. P. Pitaevskii, and S. Stringari, Rev. Mod. Phys. 80, 1215 (2008).

[24] The BCS-BEC Crossover and the Unitary Fermi Gas, Lecture Notes in Physics, edited by W. Zwerger (Springer, Heidelberg, 2012), Vol. 836.

[25] S. Nascimbène, N. Navon, K. J. Jiang, F. Chevy, and C. Salomon, Nature (London) 463, 1057 (2010).

[26] M. J. H. Ku, A. Sommer, L. W. Cheuk, and M. W. Zwierlein, Science 335, 563 (2012).

[27] I. Ferrier-Barbut, M. Delehaye, S. Laurent, A. T. Grier, M. Pierce, B. S. Rem, F. Chevy, and C. Salomon, Science 345, 1035 (2014).

[28] D. Husmann, S. Uchino, S. Krinner, M. Lebrat, T. Giamarchi, T. Esslinger, and J.-P. Brantut, Science 350, 1498 (2015).

[29] G. Valtolina, A. Burchianti, A. Amico, E. Neri, K. Xhani, J. A. Seman, A. Trombettoni, A. Smerzi, M. Zaccanti, M. Inguscio, and G. Roati, Science 350, 1505 (2015).

[30] M. J. H. Ku, B. Mukherjee, T. Yefsah, and M. W. Zwierlein, Phys. Rev. Lett. 116, 045304 (2016).

[31] M. Cetina, M. Jag, R. S. Lous, I. Fritsche, J. T. M. Walraven, R. Grimm, J. Levinsen, M. M. Parish, R. Schmidt, M. Knap, and E. Demler, Science 354, 96 (2016).

[32] J. Carlson, S. Gandolfi, and A. Gezerlis, Prog. Theor. Exp. Phys. 2012, 01A209 (2012).

[33] G. C. Strinati, P. Pieri, G. Roepke, P. Schuck, and M. Urban, Phys. Rep. 738, 1 (2018).

[34] S. Endo and Y. Castin, J. Phys. A 49, 265301 (2016).

[35] P. Nozières and S. Schmitt-Rink, J. Low Temp. Phys. 59, 195 (1985).

[36] G. C. Strinati, Lect. Notes Phys. 836, 99 (2012).

[37] R. Rossi, F. Werner, N. Prokof'ev, and B. Svistunov, Phys. Rev. B 93, 161102(R) (2016). 
[38] G. Parisi, Phys. Lett. B 66, 382 (1977).

[39] C. Itzykson, G. Parisi, and J.-B. Zuber, Phys. Rev. D 16, 996 (1977).

[40] R. Balian, C. Itzykson, J.-B. Zuber, and G. Parisi, Phys. Rev. D 17, 1041 (1978).

[41] E. B. Bogolmony and V. Fateyev, Phys. Lett. B 76, 210 (1978).

[42] M. Fry, Phys. Lett. B 80, 65 (1978).

[43] R. Rossi, Ph.D. thesis, Ecole Normale Supérieure, 2017, http://tel.archives-ouvertes.fr/tel-01704724.

[44] R. Rossi, T. Ohgoe, K. Van Houcke, and F. Werner (in preparation).

[45] F. Nevanlinna, Ann. Acad. Sci. Fenn. Ser. A1, Mat. XII, 181 (1919).

[46] J. P. Ramis, in Complex Analysis, Microlocal Calculus and Relativistic Quantum Theory, Lecture Notes in Physics, edited by D. Iagolnitzer (Springer, Berlin, Heidelberg, 1980), Vol. 126, p. 178.

[47] W. Balser, From Divergent Power Series to Analytic Functions, Lecture Notes in Mathematics (Springer, Berlin, Heidelberg, 1994), Vol. 1582.

[48] The parameters $b$ and $c$ in the Borel transform are absent from Nevanlinna's formulation, but we expect that the theorem remains valid in the presence of these parameters, since this does not change the leading large-order behavior.

[49] One of the original hypotheses in Refs. [45,47] follows from our hypothesis 2 thanks to Taylor's theorem with the Lagrange remainder $[43,46]$.

[50] For $\phi^{4}$ theory, $Q(z)$ is expected to have only one branch cut, namely the real negative axis, with a discontinuity $\sim e^{-A /|z|}$ (the corresponding branch cut for the Borel transform is $(-\infty,-A]$, as used in the conformal mapping of Ref. [5]), and in one-dimensional $\phi^{4}$ theory there is a clear physical interpretation in terms of the tunneling of a quantum particle through the barrier $x^{2}+z x^{4}$ for $z<0$, whose rate is given by the classical action $A /|z|$ [51-57]. For $\phi^{4}$ theory in 2 and 3 dimensions, Borel summability was even proven fully rigorously $[58,59]$.

[51] C. Bender and T. T. Wu, Phys. Rev. D 7, 1620 (1973).

[52] G. Parisi, Phys. Lett. B 66, 167 (1977).

[53] E. B. Bogomolny, Phys. Lett. B 67, 193 (1977).

[54] J. Zinn-Justin, Phys. Rep. 70, 109 (1981).
[55] V. Rivasseau, From Perturbative to Constructive Renormalization (Princeton University Press, Princeton, NJ, 2014).

[56] J. Zinn-Justin, Quantum Field Theory and Critical Phenomena (Clarendon Press, New York, 2002).

[57] M. Marińo, Instantons and Large N: An Introduction to Non-Perturbative Methods in Quantum Field Theory (Cambridge University Press, Cambridge, England, 2015).

[58] J.-P. Eckmann, J. Magnen, and R. Sénéor, Commun. Math. Phys. 39, 251 (1975).

[59] J. Magnen and R. Sénéor, Commun. Math. Phys. 56, 237 (1977).

[60] K. Van Houcke, F. Werner, N. Prokof'ev, and B. Svistunov, arXiv:1305.3901.

[61] Restricting to the lowest-order diagram for $\Sigma$ and $\Pi$ ( $N_{\max }=1$ in Figs. 3 and 4 ) is equivalent to the selfconsistent $T$-matrix approximation of Refs. [62,63].

[62] R. Haussmann, Phys. Rev. B 49, 12975 (1994).

[63] R. Haussmann, W. Rantner, S. Cerrito, and W. Zwerger, Phys. Rev. A 75, 023610 (2007).

[64] Here, $\Sigma(z)$ and $\Pi(z)$ have the Taylor series $\sum_{N=1}^{\infty} \Sigma^{(N)} z^{N}$ and $\sum_{N=1}^{\infty} \Pi^{(N)} z^{N}$, where $\Sigma^{(N)}$ and $\Pi^{(N)}$ are defined in Ref. [60].

[65] See Supplemental Material at http://link.aps.org/ supplemental/10.1103/PhysRevLett.121.130405, which includes Ref. [66], for data for the equation of state.

[66] T.-L. Ho, Phys. Rev. Lett. 92, 090402 (2004).

[67] K. Van Houcke, F. Werner, E. Kozik, N. Prokof'ev, B. Svistunov, M. J. H. Ku, A. T. Sommer, L. W. Cheuk, A. Schirotzek, and M. W. Zwierlein, Nat. Phys. 8, 366 (2012).

[68] T.-L. Ho and E. J. Mueller, Phys. Rev. Lett. 92, 160404 (2004).

[69] X.-J. Liu, H. Hu, and P. D. Drummond, Phys. Rev. Lett. 102, 160401 (2009).

[70] C. Gao, S. Endo, and Y. Castin, Europhys. Lett. 109, 16003 (2015).

[71] Y. Yan and D. Blume, Phys. Rev. Lett. 116, 230401 (2016).

[72] G. Parisi, Phys. Lett. B 76, 65 (1978).

[73] C. Bauer, G. S. Bali, and A. Pineda, Phys. Rev. Lett. 108, 242002 (2012).

[74] G. V. Dunne and M. Ünsal, Annu. Rev. Nucl. Part. Sci. 66, 245 (2016). 